\title{
Darstellung und Struktur von Thallium(I)-2,4,6-tris(trifluormethyl)thiophenolat, einer Verbindung mit faltblattartig-polymerem Aufbau
}

\section{Dieter Labahn, Ehmke Pohl, Regine Herbst-Irmer, Dietmar Stalke, Herbert W. Roesky* und George M. Sheldrick}

Institut für Anorganische Chemie der Universität Göttingen,

Tammannstraße 4, D-3400 Göttingen

Eingegangen am 3. Dezember 1990

Key Words: Thallium compound / Polymeric structure / Organo fluorine ligand

Preparation and Structure of Thallium(I) 2,4,6-Tris(trifluoromethyl)thiophenolate, a Compound with a Folded Polymeric Structure

The title compound, thallium(I) 2,4,6-tris(trifluoromethyl)thiophenolate (1) has been prepared by using either TlOEt and $\left(\mathrm{CF}_{3}\right)_{3} \mathrm{C}_{6} \mathrm{H}_{2} \mathrm{SH}$ or $\mathrm{Tl}_{2} \mathrm{CO}_{3}$ and $\left(\mathrm{CF}_{3}\right)_{3} \mathrm{C}_{6} \mathrm{H}_{2} \mathrm{SNa}$. A single X-ray structural analysis shows a folded polymeric structure for $\mathbf{1}$; the crystal lattice incorporates half of a 1,4-dioxane molecule per $\mathrm{Tl}$ atom. This compound has a surprisingly high kinetic stability compared to known thallium(l) thiolates.
Thallium(I)-thiolate waren in jüngster Zeit das Ziel intensiver Untersuchungen ${ }^{1-3)}$. Obwohl die ersten Vertreter schon vor über 40 Jahren dargestellt worden sind ${ }^{4)}$, konnten erst vor kurzem bei zwei Thallium(I)-thiolaten die Strukturen aufgeklärt werden ${ }^{1)}$. Dies dürfte an der schlechten Löslichkeit der polymer aufgebauten Thallium(I)-thiolate in organischen Lösungsmitteln liegen. Nachdem wir vor einiger Zeit die Synthese des $\left[\mathrm{TlOR}_{\mathrm{f}}\right]_{2}$ beschrieben haben $\left[\mathrm{R}_{\mathrm{f}}=\right.$ 2,4,6-Tris(trifluormethyl)pheny $]^{5}$, waren wir an der Darstellung der Schwefel-analogen Verbindung interessiert, um ein zweites Glied in der Reihe $\left[T_{I E R}\right]$ zu erhalten $(E=O$, $\mathrm{S}, \mathrm{Se}, \mathrm{Te}$ ). Während $\mathrm{TlOR}_{\mathrm{f}}$ im festen Zustand als Dimeres vorliegt und die Thalliumatome die niedrige Koordinationszahl 2 besitzen, ist Thallium-2,4,6-tris(trifluormethyl)thiophenolat (1) polymer aufgebaut. Es ist aber im Gegensatz zu den bisher bekannten Thallium(I)-thiolaten in stark koordinierenden Lösungsmitteln wie THF, MeCN und Dioxan sehr gut löslich, was eine Verwendung in Lösung möglich macht. So konnten durch langsames Abkühlen einer Lösung von 1 in Dioxan auf $12{ }^{\circ} \mathrm{C}$ geeignete Kristalle für eine Röntgenstrukturanalyse erhalten werden.

1 entsteht sowohl bei der Reaktion von TlOEt mit 2,4,6Tris(trifluormethyl)thiophenol in $n$-Hexan als auch bei der Umsetzung von $\mathrm{Tl}_{2} \mathrm{CO}_{3}$ mit Natrium-2,4,6-tris(trifluormethyl)thiophenolat in $\mathrm{MeCN}$ in guter Ausbeute.

$$
\begin{aligned}
& \mathrm{TIOEt}+\mathrm{R}_{\mathrm{f}} \mathrm{SH} \longrightarrow 1 / n\left[\mathrm{TISR}_{\mathrm{f}}\right]_{n}+\mathrm{EtOH} \\
& 1 \\
& \mathrm{Tl}_{2} \mathrm{CO}_{3}+2 \mathrm{R}_{\mathbf{f}} \mathrm{SNa} \longrightarrow 2 / n\left[\mathrm{TlSR}_{\mathbf{f}}\right]_{n}+\mathrm{Na}_{2} \mathrm{CO}_{3} \\
& 1
\end{aligned}
$$

1 ist ein farbloser, flockiger Feststoff, der nur wenig hydrolyseempfindlich ist und sich unter Inertgas über einen langen Zeitraum nicht zersetzt. Die bei einigen Thallium(I)thiolaten beobachtete Lichtempfindlichkeit" ${ }^{1)}$ wurde bei 1 nicht festgestellt.
Im ${ }^{1}$ H-NMR-Spektrum zeigt 1 ein Singulett $(\delta=7.9)$ für die Phenylprotonen, im ${ }^{19}$ F-NMR-Spektrum je ein Singulett für die $o-\mathrm{CF}_{3}-\mathrm{Gruppen}$ und die $p-\mathrm{CF}_{3}$-Gruppe im Intensitätsverhältnis 2:1. Im Massenspektrum (EI) findet man den Peak für die monomere Einheit TISR $\mathrm{f}_{\mathrm{f}}$ mit korrekter Isotopenverteilung.

\section{Strukturbeschreibung}

Die farblosen Kristalle sind aus Polymeren aufgebaut, deren kleinste Einheit die Summenformel TISR $\times 0.5$ Dioxan

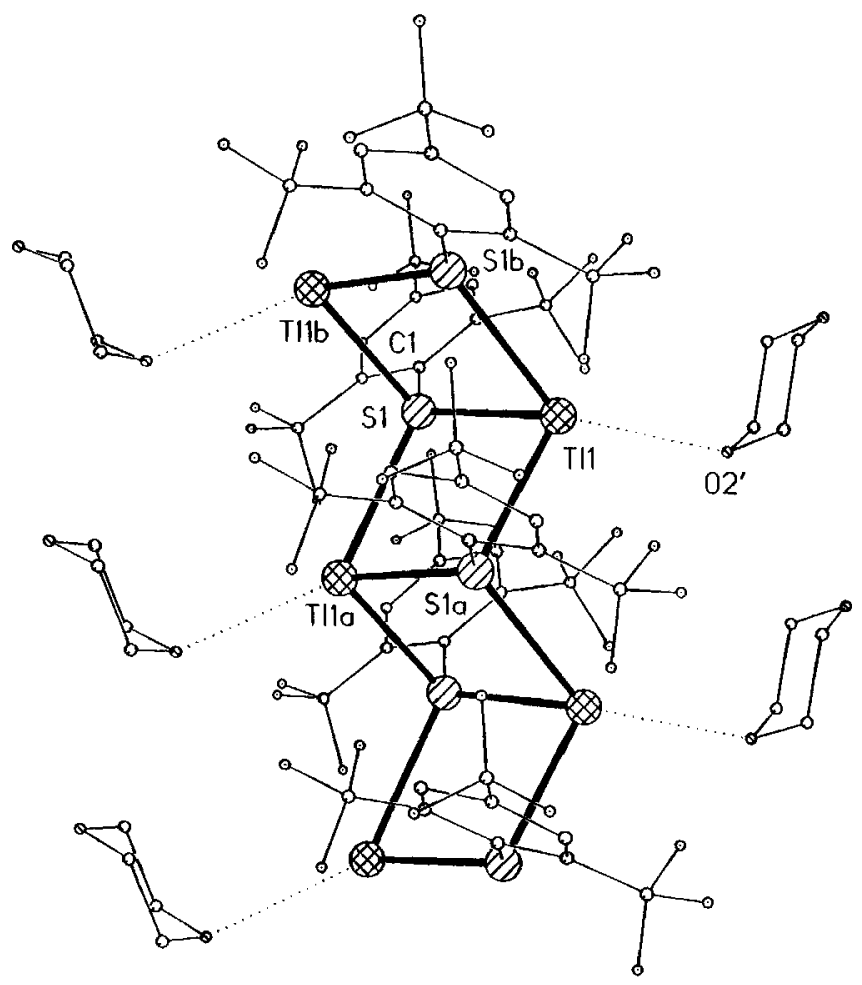

Abb. 1. Struktur von 1 mit Dioxan im Kristall 
besitzt. Die Struktur besteht aus faltblattartig angeordneten [TISR ${ }_{\mathrm{f}}$-Einheiten, die ein Band ausbilden ${ }^{\text {\%) }}$ (Abb. 1). Jedes Thalliumatom wird außer von drei Schwefelatomen im Band noch von einem Sauerstoffatom eines Dioxanmoleküls koordiniert. Jedes Dioxanmolekül verbrückt zwei benachbarte Bänder. Die Dioxanmoleküle füllen so die Lücken zwischen den Bändern.

Die anellierten viergliedrigen Ringe besitzen eine gemeinsame Tl-S-Bindung von 311.7 pm [Tl(1)-S(1)] (Tab. 2). Die beiden anderen $\mathrm{Tl}-\mathrm{S}$-Abstände innerhalb der viergliedrigen Ringe betragen 300.7 [Tl(1)-S(1A)] und $324.2 \mathrm{pm}[\mathrm{Tl}(1)-$ $S(1 B)]$ und sind vergleichbar mit denen in ähnlichen Thallium(I)-Oligomeren ${ }^{1)}$. Die Flächennormalen zweier benachbarter viergliedriger Ringe bilden einen Winkel von $106.5^{\circ}$.

Diese Arbeit wurde von der Deutschen Forschungsgemeinschaft, dem Fonds der Chemischen Industrie und der Stiftung Volkswagenwerk gefördert.

\section{Experimenteller Teil}

Ausgangsmaterialien: 2,4,6-Tris(trifluormethyl)thiophenol wurde nach Lit. ${ }^{7)}$ dargestellt. Die Umsetzung mit $\mathrm{NaH}$ ergab das Natrium2,4,6-tris(trifluormethyl)thiophenolat. Alle verwendeten Lösungsmittel wurden nach bekannten Verfahren getrocknet und von Sauerstoff befreit.

Thallium(I)-2,4,6-tris (trifluormethyl) thiophenolat (1): a) $2.0 \mathrm{~g}$ $(8 \mathrm{mmol})$ Thallium(I)-ethanolat werden in $20 \mathrm{ml} n$-Hexan vorgelegt und tropfenweise mit einer Lösung von $2.5 \mathrm{~g} \mathrm{(} 8 \mathrm{mmol})$ 2,4,6-Tris(trifluormethyl)thiophenol in $20 \mathrm{ml} n$-Hexan versetzt. Man läßt $16 \mathrm{~h}$ bei Raumtemp. rühren und filtriert ausgefallenes $1 \mathrm{ab}$. Im Ölpumpenvak. wird von anhaftenden flüchtigen Bestandteilen befreit: farbloser, flockiger Feststoff 1 wird in 87 proz. Ausb. (3.6 g) erhalten und schmilzt bei $230^{\circ} \mathrm{C}$ unter Zersetzung.

b) $2.0 \mathrm{~g}(6 \mathrm{mmol}) \mathrm{R}_{\mathrm{f}} \mathrm{SNa}$ und $1.4 \mathrm{~g}(3 \mathrm{mmol}) \mathrm{Tl}_{2} \mathrm{CO}_{3}$ werden mit $40 \mathrm{ml} \mathrm{MeCN}$ versetzt und die Reaktionslösung wird $2 \mathrm{~d}$ bei Raumtemp. gerühtt. Man engt die Lösung auf die Hälfte ein und filtriert die unlöslichen Anteile über eine dünne Schicht Celite. Nach Einengen des Filtrats erhält man $2.5 \mathrm{~g}(80 \%)$ 1. - ${ }^{\mathrm{t}} \mathrm{H}-\mathrm{NMR}(80.13$ $\mathrm{MHz}$, ext. TMS, $\mathrm{CD}_{3} \mathrm{CN}$ ): $\delta=7.9$ (s). $-{ }^{19} \mathrm{~F}$-NMR $(75.39 \mathrm{MHz}$, ext. $\left.\mathrm{CFCl}_{3}, \mathrm{CD}_{3} \mathrm{CN}\right): \delta=-60.9\left(\mathrm{~s}, 6 \mathrm{~F}, o-\mathrm{CF}_{3}\right),-61.4$ (s, 3F, p$\mathrm{CF}_{3}$ ). - IR (Nujol, CsI): $\tilde{v}=2955 \mathrm{~cm}^{-1}$ sst, 2925 sst, 2855 sst, 1461 st, 1377 st, 1291 st, 1190 st, 1123 st, 1098 st, 918 st, 254 st. $\operatorname{MS}(\mathrm{EI}): m / z(\%)=626\left[\left(\mathrm{R}_{\mathrm{f}} \mathrm{S}\right)_{2}^{+}\right](1), 518\left[\mathrm{TlSR}_{\mathrm{f}}^{+}\right](6), 294\left[\mathrm{R}_{\mathrm{f}} \mathrm{S}^{+}-\right.$ $\mathrm{F}](17), 205\left[\mathrm{Tl}^{+}\right](100), 69\left[\mathrm{CF}_{3}^{+}\right](6)$; korrekte Isotopenverteilung.

$$
\begin{array}{lll}
\mathrm{C}_{9} \mathrm{H}_{2} \mathrm{~F}_{9} \mathrm{STl} \text { (517.5) } & \text { Ber. C } 20.89 \text { H } 0.39 \\
& \text { Gef. C } 20.7 \text { H } 0.34
\end{array}
$$

Kristallstrukturanalyse von 1: Für die Datensammlung wurde cin $0.2 \times 0.2 \times 0.4 \mathrm{~mm}$ großer Einkristall in einem inerten Öl auf einen Glasfaden montiert und im Kaltgasstrom bei $-120^{\circ} \mathrm{C}$ schockgefroren. Die Datensammlung erfolgte auf einem Stoe-Siemens-AED-Vierkreisdiffraktometer mit graphitmonochromatisierter Mo- $K_{\alpha}$-Strahlung $(\lambda=71.073 \mathrm{pm})$. Es wurde eine empirische Absorptionskorrektur mit 421 Reflexmessungen bei verschiedenen $\psi$-Winkeln durchgeführt, max. und min. Transmission 0.435 und 0.138. Die Struktur wurde mit Patterson- und Fourier-Methoden gelöst ${ }^{83}$. Sämtliche Nichtwasserstoffatome wurden anisotrop verfeinert. Die Wasserstoffatome wurden geometrisch ideal positioniert $\left[\mathrm{C}-\mathrm{H}\right.$-Abstand $96 \mathrm{pm}, U(\mathrm{H})=400$ bzw. $\left.500 \mathrm{pm}^{2}\right]$ und nach einem Reitermodell verfcinert. Die $p$-ständige $\mathrm{CF}_{3}$-Gruppe ist rotationsfehlgeordnet. Zwei Positionen (F41 - F43 und F411 - F431) wurden mit einem Besetzungsfaktor von 0.5 und Abstands-Restraints verfeinert (SHELX-76, vom Autor verbesserte Version).

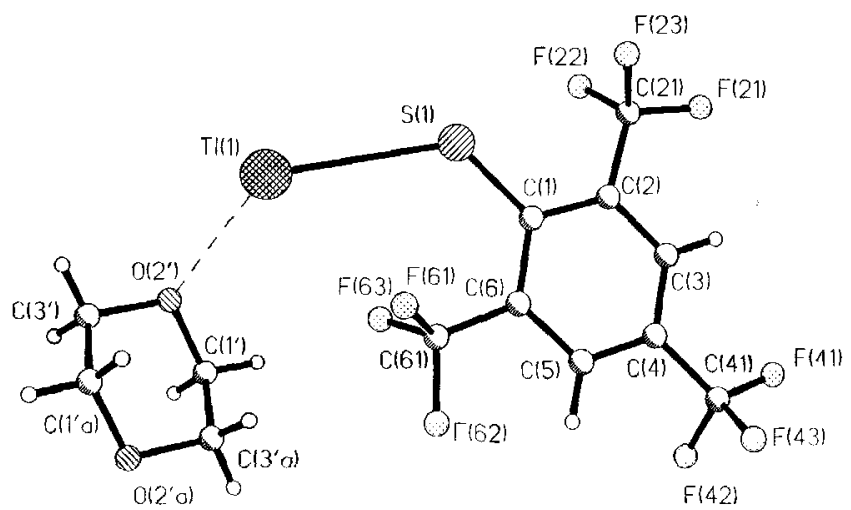

Abb. 2. Struktur von 1 mit Atomkennzeichnung

Tab. 1. Atomkoordination $\left[\times 10^{4}\right]$ und $U_{\mathrm{eq}}$-Werte $\left[\mathrm{pm}^{2} \times 10^{-1}\right]$ von $1\left(U_{\mathrm{eq}}\right.$ berechnet als ein Drittel der Spur des orthogonalen $U_{i j}$-Tensors)

\begin{tabular}{|c|c|c|c|c|}
\hline & $\mathrm{x}$ & $\mathrm{y}$ & $z$ & $\mathrm{U}(\mathrm{eq})$ \\
\hline$I I(1)$ & $2968(1)$ & $4754(1)$ & $3445(1)$ & $24(1)$ \\
\hline$S(1)$ & $3333(1)$ & $5056(3)$ & $2109(1)$ & $23(1)$ \\
\hline$C(I)$ & $4381(4)$ & $6431(12)$ & $1900(3)$ & $18(2)$ \\
\hline$c(2)$ & $4528(5)$ & $6129(14)$ & $1291(3)$ & $23(2)$ \\
\hline$c(21)$ & $3810(6)$ & $4558(15)$ & $799(3)$ & $28(2)$ \\
\hline$F(21)$ & $4082(4)$ & $4551(12)$ & $255(2)$ & $48(2)$ \\
\hline$F(22)$ & $3717(3)$ & $1937(9)$ & $951(2)$ & $37(1)$ \\
\hline$F(23)$ & $2876(3)$ & $5577(10)$ & $662(2)$ & $38(1)$ \\
\hline$c(3)$ & $5345(5)$ & $7312(14)$ & $1117(3)$ & $28(2)$ \\
\hline$c(4)$ & $6027(5)$ & $8821(16)$ & $1536(3)$ & $31(2)$ \\
\hline$C(41)$ & $6902(6)$ & $10085(14)$ & $1354(4)$ & $44(3)$ \\
\hline$F(4 I)$ & $7083(14)$ & $9243(55)$ & $843(7)$ & $146(15)$ \\
\hline$F(42)$ & $7723(8)$ & $9451(38)$ & $1759(8)$ & $80(7)$ \\
\hline$F(43)$ & $6903(12)$ & $12618(20)$ & $1334(12)$ & $107(13)$ \\
\hline$F(411)$ & $7216(18)$ & $12198(36)$ & $1673(9)$ & $115(12)$ \\
\hline$F(421)$ & $6624(13)$ & $11026(51)$ & $793(6)$ & $102(10)$ \\
\hline$F(431)$ & $7644(14)$ & $8621(33)$ & $1360(14)$ & $203(24)$ \\
\hline$C(5)$ & $5919(5)$ & $9137(15)$ & $2136(3)$ & $30(2)$ \\
\hline$c(6)$ & $5113(5)$ & $7949(13)$ & $2323(3)$ & $23(2)$ \\
\hline$c(61)$ & $5080(5)$ & $8290(14)$ & $2990(3)$ & $27(2)$ \\
\hline$F(61)$ & $4249(3)$ & $9541(9)$ & $3070(2)$ & $32(1)$ \\
\hline$F(62)$ & $5846(4)$ & $9757(10)$ & $3314(2)$ & $42(2)$ \\
\hline$E(63)$ & $5123(3)$ & $5895(9)$ & $3286(2)$ & $31(1)$ \\
\hline$C\left(1^{\prime}\right)$ & $5559(7)$ & $3418(15)$ & $4691(3)$ & $36(2)$ \\
\hline$O\left(2^{\prime}\right)$ & $4529(4)$ & $2867(10)$ & $4606(2)$ & $35(2)$ \\
\hline$c\left(3^{\circ}\right)$ & $4203(7)$ & $3630(15)$ & $5160(3)$ & $36(3)$ \\
\hline
\end{tabular}

Tab. 2. Ausgewählte Bindungsabstände $[\mathrm{pm}]$ und -winkel $\left[{ }^{\circ}\right]$ in $\mathbf{1}$

\begin{tabular}{|c|c|c|c|c|c|}
\hline $\begin{array}{l}\operatorname{Tl}(1)-S(1) \\
\operatorname{Il}(1)-S(1 B) \\
\operatorname{Tl}(1)-O\left(2^{\prime}\right)\end{array}$ & $\begin{array}{l}311 . \\
324 . \\
307 .\end{array}$ & $\begin{array}{l}(2) \\
(2) \\
(4)\end{array}$ & $\begin{array}{l}T 1(1)-S(1 A) \\
S(1)-C(1)\end{array}$ & $\begin{array}{l}300 . \\
175\end{array}$ & $\begin{array}{l}(2) \\
(6)\end{array}$ \\
\hline \multicolumn{2}{|c|}{$\begin{array}{l}S(1)-T 1(1)-S(I A) \\
S(1 A)-T 1(1)-S(1 B) \\
T 1(1)-S(1)-T 1(1 B) \\
S(1)-T I(1)-O\left(2^{\prime}\right) \\
S(1 B)-T I(1)-O\left(2^{\prime}\right)\end{array}$} & $\begin{array}{r}82.2(1) \\
104.2(1) \\
102.0(1) \\
124.6(1) \\
140.7(1)\end{array}$ & \multicolumn{2}{|c|}{$\begin{array}{l}S(1)-T 1(1)-S(1 B) \\
T 1(1)-S(1)-T I(1 A) \\
T 1(1 A)-S(1)-T 1(I B) \\
S(1 A)-T 1(1)-O\left(2^{\prime}\right)\end{array}$} & $\begin{array}{r}78.6(1) \\
96.9(1) \\
104.2(1) \\
109.8(1)\end{array}$ \\
\hline
\end{tabular}

Kristallographische Daten: Raumgruppe $P 2_{1} / n ; a=1378.0(2)$, $b=493.2(1), c=2211.6(4) \mathrm{pm}, \beta=103.73(1)^{\circ}, V=1.460(6) \mathrm{nm}^{3}$, $Z=4, \varrho_{\text {ber }}=2.56 \mathrm{Mgm}^{-3}, \mu\left(\mathrm{Mo}-K_{\alpha}\right)=11.41 \mathrm{~mm}^{-1}, 5430 \mathrm{ge}-$ messene Reflexe, davon 4232 unabhängige und 3594 beobachtete mit $F>4 \sigma(F), 8^{\circ}<2 \Theta<60^{\circ}$. Verfcinerung mit 237 Parametern 
auf $R=0.045, R_{W}=0.047, w^{-1}=\sigma^{2}(F)+0.0005 F^{2}$, maximale und minimale Restelektronendichte: $2.93 \cdot 10^{-6}$ bzw. $-1.62 \cdot 10^{-6}$ $\mathrm{e}^{-} \mathrm{pm}^{-3}$. - Atomkoordinaten, ausgewählte Abstände und Winkel sind in Tab. 1 und 2 aufgelistet.

Weitere Einzelheiten zu den Kristallstrukturuntersuchungen können beim Fachinformationszentrum Karlsruhe, Gesellschaft für wissenschaftlich-technische Information $\mathrm{mbH}, \mathrm{D}-7514$ EggensteinLeopoldshafen 2, unter Angabe der Hinterlegungsnummer CSD320188, der Autorennamen und des Zeitschriftenzitats angefordert werden.

CAS-Registry-Nummer

1. $1 / 2 \mathrm{C}_{4} \mathrm{H}_{8} \mathrm{O}_{2}: 131703-94-1$
1) B. Krebs, A. Brömmelhaus, Angew. Chem. 101 (1989) 1726; Angew. Chem. Int. Ed. Engl. 28 (1989) 1682.

2) J. H. Green, R. Kumar, N. Seudeal, D. G. Tuck, Inorg. Chem. 28 (1989) 123.

3) R. Kumar, H. E. Mabrouk, D. G. Tuck, J. Chem. Soc., Dalton Trans. 1988, 1045.

4) H. Gilman, R. K. Abbott, J. Am. Chem. Soc. 71 (1949) 659.

${ }^{5)}$ H. W. Roesky, M. Scholz, M. Noltemeyer, F. T. Edelmann, Inorg. Chem. 28 (1989) 3829.

${ }^{6}$ Eine ähnliche Bandstruktur liegt im $\left[\mathrm{KSR}_{\mathrm{f}}\right]_{n}$ vor: S. A. Brooker, F. T. Edelmann, T. Kottke, D. Stalke, J. Chem. Soc., Chem. Commun., i. Druck.

7) G. E. Carr, R. D. Chambers, T. F. Holmes, D. G. Parker, J. Organomet. Chem. 325 (1987) 13.

8) G. M. Sheldrick, Acta Crystallogr., Sect. A. 46 (1990) 467.

[401/90] 